\title{
Evaluación del Rendimiento, Composición y Actividad Antioxidante de Aceite de Semillas de Mora (Rubus glaucus) Extraído con $\mathrm{CO}_{2}$ Supercrítico
}

\author{
Ana L. Pantoja-Chamorro(1), Andrés M. Hurtado-Benavides ${ }^{(1) \star}$ y Hugo A. Martínez-Correa ${ }^{(2)}$ \\ (1) Grupo de Investigación Tecnologías Emergentes en Agroindustria-TEA, Facultad de Ingeniería \\ Agroindustrial, Universidad de Nariño, Pasto, Colombia (e-mail: analucia_pantoja@yahoo.es; \\ ahurtadob@hotmail.com) \\ (2) Grupo de Investigación en Procesos Agroindustriales-GIPA, Universidad Nacional de Colombia, \\ Palmira-Colombia (e-mail: hamartinezco@unal.edu.co)
}

${ }^{*}$ Autor a quien debe ser dirigida la correspondencia

Recibido Abr. 26, 2016; Aceptado Jun. 15, 2016; Versión final Ago. 14, 2016, Publicado Feb. 2017

\begin{abstract}
Resumen
En este trabajo se estudió el efecto de los parámetros de extracción del aceite de semillas de mora (Rubus glaucus), utilizando $\mathrm{CO}_{2}$ supercrítico como solvente. Se evaluaron diferentes condiciones de presión y temperatura, que permitieron analizar el rendimiento, la composición y la actividad antioxidante del producto extraído. La identificación de los componentes del aceite se realizó a través de cromatografía de gases y la actividad antioxidante se determinó aplicando el método de ABTS. El rendimiento más alto fue $14.5 \%$, obtenido bajo las mejores condiciones de trabajo: 350 bar de presión y $60^{\circ} \mathrm{C}$ de temperatura. El aceite proveniente de semillas de $R$. glaucus se caracteriza por contener un $96 \%$ de ácidos grasos insaturados, de los cuales el ácido linoléico es el más abundante $(61.6 \%)$ y por la presencia de sustancias como $\beta$-sitosterol, 24-metil-cicloartanol, $\alpha$-tocoferol, $\gamma$-tocoferol, $\delta$-tocoferol, además de una capacidad antioxidante equivalente al trolox de $604 \mu \mathrm{mol} / 100 \mathrm{~g}$ aceite.
\end{abstract}

Palabras clave: aceite; actividad antioxidante; $\mathrm{CO}_{2}$ supercrítico; mora; Rubus glaucus

\section{Evaluation of the Yield, Composition and Antioxidant Activity of Blackberry (Rubus glaucus) Seed Oil Extracted with Supercritical $\mathrm{CO}_{2}$}

\begin{abstract}
The yield, composition and antioxidant activity of the extract product at different conditions of pressure and temperature were evaluated. The identification of oil components was performed using gas chromatography and the antioxidant activity was determined using the ABTS method. The highest yield obtained was $14.5 \%$ under the best working conditions: 350 bar of pressure and temperature of $60^{\circ} \mathrm{C}$. The oil from R. glaucus seed contains $96 \%$ of unsaturated fatty acids, of which linoleic acid is the most abundant $(61.6 \%)$ and the presence of substances such as $\beta$-sitosterol, 24-methyl-cycloartenol, $\alpha$-tocopherol, $\gamma$-tocopherol, $\delta$ tocopherol, and antioxidant trolox equivalent capacity of $604 \mu \mathrm{mol} / 100 \mathrm{~g}$ oil.
\end{abstract}

Keywords: oil; antioxidant activity; supercritical $\mathrm{CO}_{2}$; blackberry; Rubus glaucus 


\section{INTRODUCCIÓN}

De acuerdo a la encuesta nacional agropecuaria (año 2013), la producción de mora (Rubus glaucus) en Colombia se evaluó en 58.923 toneladas. En el mismo año, el departamento de Nariño, aparece como el tercer mayor productor con $5.677,5$ toneladas, lo cual representa el $9.6 \%$ de la producción nacional. No obstante, las empresas transformadoras de la mora centran su mayor esfuerzo en la utilización de la pulpa para la obtención de productos como jugos, pulpas, mermeladas, conservas, confites y colorantes los cuales generan valor agregado. Tras el proceso de obtención de pulpas de la fruta resultan aproximadamente 0.1 $\mathrm{kg}$ de residuos por $\mathrm{kg}$ de fruta procesada (CORPOICA, 2007). El 70\% de estos subproductos agroindustriales están representados en semillas y un $30 \%$ en restos de pulpa. A lo anterior se adiciona un $30 \%$ en pérdidas poscosecha (Ruiz, et al., 2009). La situación se agrava si se tiene en cuenta que la industria no cuenta con el conocimiento e infraestructura para dar un mayor valor agregado a la fruta, y los residuos tienen una mala disposición final o bien se destinan a alimentación para animales o al proceso de compostaje para luego ser usado como abono. El desaprovechamiento integral de la fruta es entonces evidente, además del perjuicio ocasionado a la cadena frutícola y al medio ambiente.

Diversos estudios presentan como alternativa remediadora, obtener aceite a partir de las semillas del fruto de mora, con posibles aplicaciones en la industria alimentaria, cosmética o farmacéutica por su interesante composición química, lo que permitiría convertir al subproducto en materia prima valiosa (Cerón, et al., 2012; Conrado, 2008; Van Hoed et al., 2009; García et al., 2003). Diversos autores sostienen que, dependiendo de la técnica de extracción, tipo de solvente utilizado y condiciones de operación aplicadas, el rendimiento de aceite proveniente de las semillas de mora, puede oscilar entre 9,2 y 18,3\% (Pasquel et al., 2014; García et al., 2003). El producto es rico en ácidos grasos insaturados como saturados (Micic et al., 2015; Cerón, et al., 2012), cuenta con un alto contenido de tocoferoles y esteroles (Van Hoed et al., 2009) y además, posee actividad antioxidante que incrementa su potencial industrial (Yang et al., 2011).

Existen diferentes métodos de obtención del aceite, entre los cuales se encuentran la extracción con solventes, la destilación y la extracción por prensado (Pasquel et al., 2014; Fazio et al., 2013; Cerón, et al., 2012; García et al., 2003). La destilación resulta poco conveniente para usarse con aceites vegetales debido a la presencia de triglicéridos que pueden romperse a altas temperaturas de operación necesarias. La presión en frio solo da lugar a una recuperación parcial del aceite. La extracción con solventes, particularmente con hexano deja un residuo inherente en el aceite, además de ser un solvente inflamable y tóxico, puede generar un deterioro de los ácidos grasos poliinsaturados, así como también puede provocar rancidez oxidativa, producción de sabores y olores indeseables.

Recientemente, la extracción con fluidos supercríticos se ha posicionado entre las tecnologías verdes de mayor importancia por cuanto genera extractos libres de solventes, (Al-Otoom et al., 2014), evita la degradación de componentes bioactivos y utiliza solventes no derivados de la industria petroquímica, como es el dióxido de carbono $\left(\mathrm{CO}_{2}\right)$. El $\mathrm{CO}_{2}$ es un fluido con propiedades fisicoquímicas y características ideales que permite preservar los principios activos y las características propias de los extractos debido a que alcanza su presión y temperatura crítica a 73,8 bar y $31,1^{\circ} \mathrm{C}$, además es un elemento selectivo, no inflamable, no toxico, no corrosivo, de bajo costo, naturalmente abundante y no genera residuos que afecten la salud y el medio ambiente (Norhuda y Jusoff, 2009; Nivia et al., 2007; Hurtado, 2002). Sin embargo una de las desventajas de la extracción con $\mathrm{CO}_{2}$ supercrítico es su carácter apolar, que limita su poder de solvatación de compuestos apolares.

La variación de condiciones de presión y temperatura permite obtener extractos de diferente composición. Por ejemplo Cavero, et al. (2005) reportó que del romero (Rosmarinus officinalis) es posible obtener una fracción rica en aceites esenciales en condiciones cercanas al punto crítico, y otra fracción oleoresinosa rica en antioxidantes. Además Cerón, Hurtado y Ayala (2016) reportan que al modificar la presión y la temperatura de extracción varía el rendimiento y composición del aceite de semillas de guayaba, generando varias fracciones con diferente composición; similares resultados presentaron Castro, Rodriguez y Parada (2001).

La extracción con fluidos supercríticos, permite la obtención de aceites vegetales con características particulares o fracciones con determinados componentes de interés para fines específicos, dependiendo de las condiciones de presión y temperatura de extracción. El objetivo del presente trabajo fue evaluar los efectos de los parámetros de extracción sobre el rendimiento, la composición de ingredientes activos tales como tocoferoles, esteroles y ácidos grasos y la determinación de la actividad antioxidante del aceite de semillas de mora. 


\section{MATERIALES Y METODOS}

Se presenta la preparación de la muestra, la extracción con fluidos supercríticos de aceite de semillas de mora, el análisis de ácidos grasos, el análisis de esteroles y tocoferoles, la determinación de actividad antioxidante mediante el método radical ABTS y finalmente se presenta el análisis estadístico.

\section{Preparación de la muestra}

Las semillas de mora utilizadas como fuente de aceite, fueron suministradas por la empresa Industria de Productos Agroindustriales de Nariño (INPADENA), en el municipio de Pasto (Nariño). Posterior al proceso de lavado, el material se colocó en un secador de bandejas (FIQ, Colombia) a $60^{\circ} \mathrm{C}$ durante 8 horas hasta un porcentaje de humedad remanente de $7,60 \%$ (base seca); seguidamente se empacó en bolsas de papel kraft y se almacenó a temperatura ambiente. El producto seco se llevó a un molino de cuchillas (IKA A11 Basic, Alemania) obteniendo un Dp medio de 727,3 $\mu \mathrm{m}$, se empacó al vacío y almacenó en refrigeración a $4^{\circ} \mathrm{C}$, hasta su posterior utilización.

\section{Extracción del aceite}

El aceite de semillas de mora se obtuvo mediante la aplicación de la tecnología de extracción con fluidos supercríticos bajo diferentes condiciones de presión y temperatura. Para lograrlo, se manejó un equipo Waters SFE-500 (USA), como se muestra en el diagrama de proceso (Figura 1), y se utilizó $\mathrm{CO}_{2}$ al 99,9\% de pureza (Cryogas, Colombia) como solvente. El proceso extractivo se llevó a cabo utilizando una carga de $250 \mathrm{~g}$ de semillas tratadas, intervalos de presión de 200 a 350 bares, temperaturas entre 40 y $60^{\circ} \mathrm{C}$, manteniendo un flujo constante de $30 \mathrm{~g} \mathrm{CO}_{2} / \mathrm{min}$ y un tiempo de $150 \mathrm{~min}$. Estos valores se determinaron basándose en la cinética del proceso y estudios preliminares de ésta investigación. Se aplicó un diseño factorial $2^{2}$ con 4 puntos centrales por duplicado y de manera aleatoria, de acuerdo a la metodología descrita por Hurtado-Benavides, Dorado y Sanchez-Camargo (2016), con algunas modificaciones. El aceite obtenido se colectó y almacenó en un vial de vidrio ámbar en refrigeración $\left(4^{\circ} \mathrm{C}\right)$, hasta su posterior análisis. El rendimiento fue calculado mediante la ecuación 1, y se expresó como porcentaje de aceite extraído.

$\% R A=\left(\frac{P A}{P S}\right) \times 100$

Donde: \%RA: Porcentaje de rendimiento de aceite extraído; PA, peso del aceite extraído (g); PS, peso total de semilla utilizada para cada extracción (g).

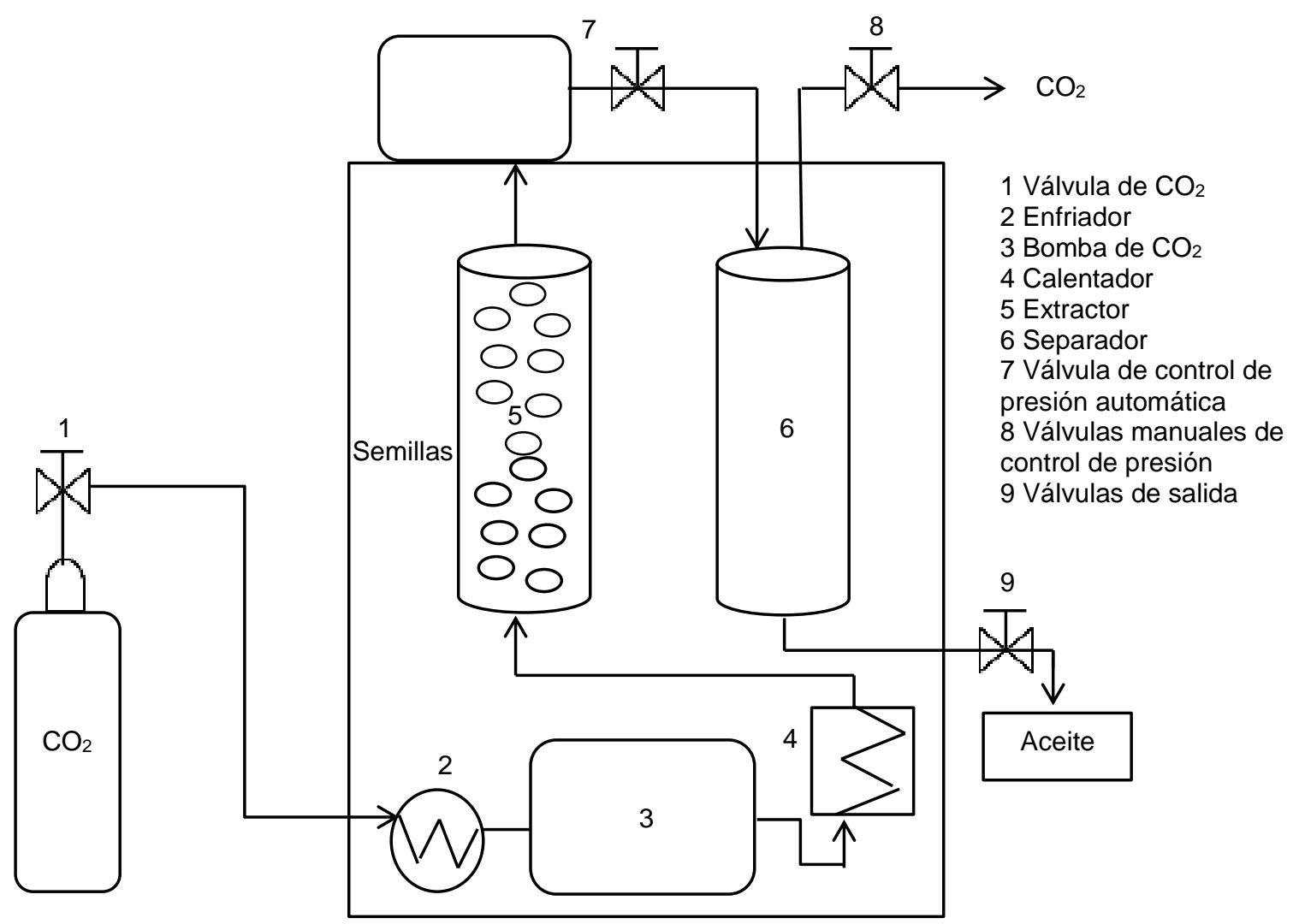

Fig. 1: Diagrama de proceso de extracción con fluidos supercríticos 


\section{Composición de ácidos grasos}

Los ácidos grasos del aceite fueron analizados como metil ésteres de ácidos grasos empleando la metodología descrita por Christie W. (1998) con algunas modificaciones. $200 \mathrm{mg}$ de aceite se derivatizaron con una solución de $\mathrm{HCl} /$ metanol al $5 \%$ a $50^{\circ} \mathrm{C}$. La extracción de los metil ésteres de los ácidos grasos se realizó con $2 \mathrm{~mL}$ de n-hexano grado HPLC (Fisher, USA). Se inyectó $1 \mu \mathrm{L}$ de la muestra a una temperatura de $250^{\circ} \mathrm{C}$, con un flujo de He de $1 \mathrm{~mL} / \mathrm{min}$. Para el análisis cromatográfico se utilizó un cromatógrafo de gases (Shimadzu GC 17A, Japón), equipado con un detector de ionización de llama (FID a $280^{\circ} \mathrm{C}$ ), y modo de inyección Split 1:10. Los compuestos fueron separados en una columna DB-WAX (JyW Scientific) de 30 $\mathrm{m}$ de largo, con diámetro interno de 0,25 $\mathrm{mm}$ y espesor de película de 0,25 $\mu \mathrm{m}$. Los ácidos grasos fueron identificados por comparación de sus tiempos de retención con aquellos de una mezcla estándar de ácidos grasos (Restek, USA) analizados bajo las mismas condiciones operacionales. La cuantificación se realizó por porcentaje relativo de área y estándar interno empleando metil undecanoato (Alltech, USA).

\section{Composición de esteroles y tocoferoles}

La composición de esteroles y tocoferoles se determinó según la metodología descrita por Matthaus y Ozcan (2011) con algunas modificaciones. Se utilizaron $100 \mu \mathrm{L}$ de aceite se diluyeron a $1 \mathrm{~mL}$ con diclorometano grado HPLC (Honeywell, USA). Los extractos se agitaron en un vortex (0,5 min), y se les adicionó sulfato de sodio anhidro (Merck, Germany). Se inyectó 1,0 $\mu \mathrm{L}$ en un cromatógrafo de gases acoplado a espectrometría de masas (Shimadzu QP2010S, USA), equipado con una columna SHXRI-5MS (Shimadzu. $30 \mathrm{~m} \times 0,25 \mathrm{~mm} \times 0,25 \mu \mathrm{m}$ ), un detector selectivo de masas QP2010S con ionización por impacto electrónico (full scan $\mathrm{El} \mathrm{a} 70 \mathrm{eV}$ ) y un inyector en modo de inyección splitless a $270^{\circ} \mathrm{C}$. La identificación tentativa de los compuestos del aceite de mora se realizó por comparación de los espectros de masas, empleando la base de datos NIST. La cuantificación se llevó a cabo utilizando como estándar una solución de patrón de Colesterol (Sigma, USA).

\section{Actividad antioxidante. Método ABTS*}

La metodología aplicada fue la descrita por Re et al. (1999), citado por Jimenez y Villareal (2008). El radical $\mathrm{ABTS}^{\circ+}$ se generó tras la reacción de ABTS $(7 \mathrm{mM})$ con persulfato potásico $(2,45 \mathrm{mM})$. La Mezcla reaccionante permaneció a temperatura ambiente y en ausencia de luz durante 24 horas. El ABTS ${ }^{\circ+}$ preparado se diluyó con etanol hasta obtener un valor de absorbancia de 0,7 $\pm 0,2$ a una longitud de onda de $734 \mathrm{~nm}$, y utilizando un espectrofotómetro Genesys UV-VIS 10S (Thermo Scientific, USA). El potencial antioxidante de la muestra se probó por la adición de $1 \mathrm{~mL}$ de la solución del radical $\mathrm{ABTS}^{{ }^{+}}$a $10 \mu \mathrm{L}$ del aceite diluido en una mezcla isopropanol: etanol (80:20) y se leyó la absorbancia a $734 \mathrm{~nm}$; la lectura se interpoló en la ecuación de la recta obtenida a partir de trolox $(0,05-1,5 \mathrm{mM})$. Para la mezcla reaccionante se utilizaron $30 \mu \mathrm{L}$ de solución de trolox y $3 \mathrm{~mL}$ de $\mathrm{ABTS}^{\circ+}$. En cada ensayo se aplicó 1 min de agitación y 6 min de reposo, al final de los cuales se tomó la lectura de $A_{734}$. Cada prueba se realizó por triplicado; la ecuación de la recta fue $\mathrm{y}=0,8885-0,3102 X, R^{2}=0,9979$.

\section{Análisis estadístico}

Con el fin de determinar el efecto de la presión y la temperatura sobre el rendimiento, la composición y el potencial antioxidante del aceite, se realizó un análisis de varianza (ANOVA, $P<0,05$ ) con ayuda del programa estadístico STATGRAPHICS Centurion XV (2007). Las determinaciones analíticas y experimentos fueron realizados por duplicado.

\section{RESULTADOS Y DISCUSIÓN}

\section{Efecto de la presión y la temperatura de extracción sobre el rendimiento}

En la figura 2 se muestra el efecto de la presión y temperatura de extracción frente al porcentaje de rendimiento de aceite de semillas de mora. Puede observarse que cuando se lleva a cabo la extracción en el nivel bajo de presión (200 bar), el rendimiento es menor; mientras que al trabajar en los niveles altos de presión y temperatura $\left(350\right.$ bar- $\left.60^{\circ} \mathrm{C}\right)$, el rendimiento de aceite se incrementa notablemente. El mayor rendimiento fue del $14,5 \%$ de aceite.

La figura 3 ilustra que la presión tiene mayor influencia que la temperatura en el rendimiento de aceite. La pendiente muestra un efecto positivo de la presión sobre el rendimiento, que se produce por el aumento de la densidad del $\mathrm{CO}_{2}$ con el incremento de la presión, y aumento de la solubilidad del aceite en el disolvente. Este efecto ha sido descrito por otros investigadores (Liu, Xu et al., 2012; Duba y Fiori, 2015). 
El efecto significativo de la interacción presión-temperatura sobre el rendimiento se observa en la figura 4, la cual presenta que a 200 bar de presión, la temperatura no ejerce un efecto significativo sobre el rendimiento, al pasar de 40 a $60^{\circ} \mathrm{C}$ el rendimiento tuvo solo un incremento alrededor del $2 \%$; mientras que a 350 bar, la temperatura presenta diferencias estadísticas en el rendimiento de aceite de mora.

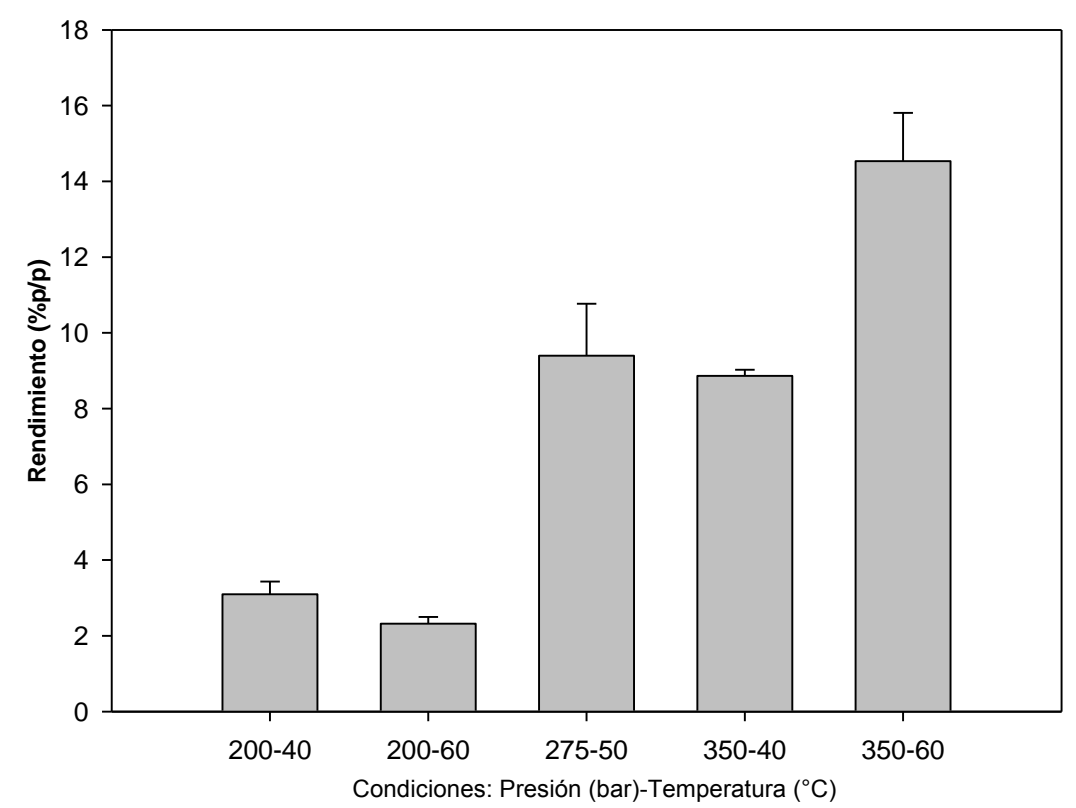

Fig. 2: Resultados del rendimiento de extracción de aceite de semillas de mora (\%p/p)

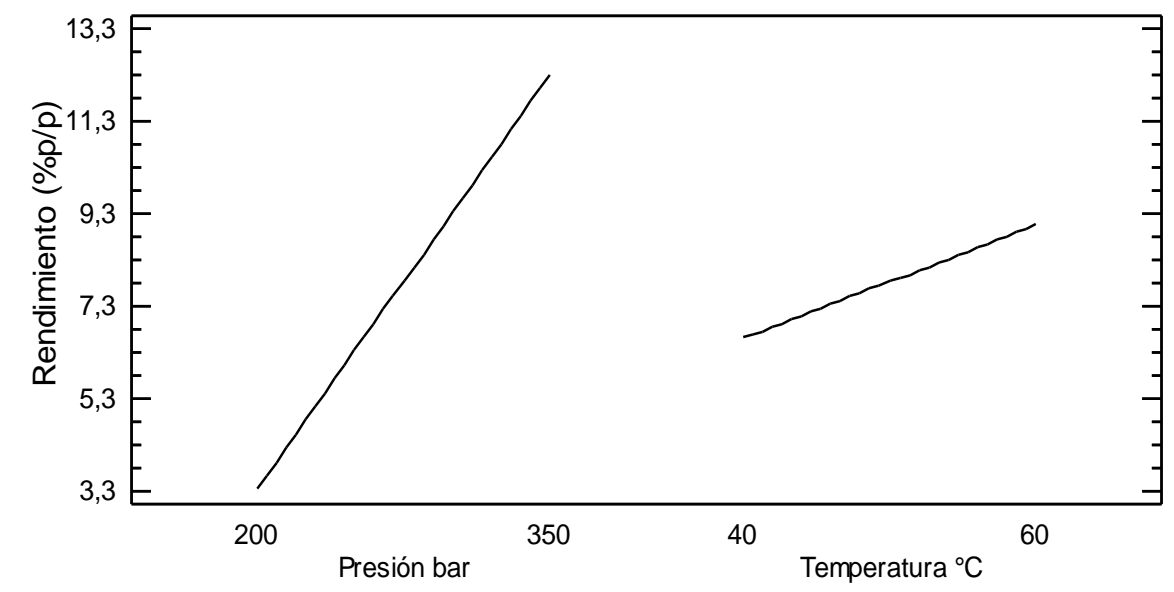

Fig. 3: Diagrama de efectos principales para rendimiento en la extracción de aceite de mora con $\mathrm{CO}_{2}$ supercrítico

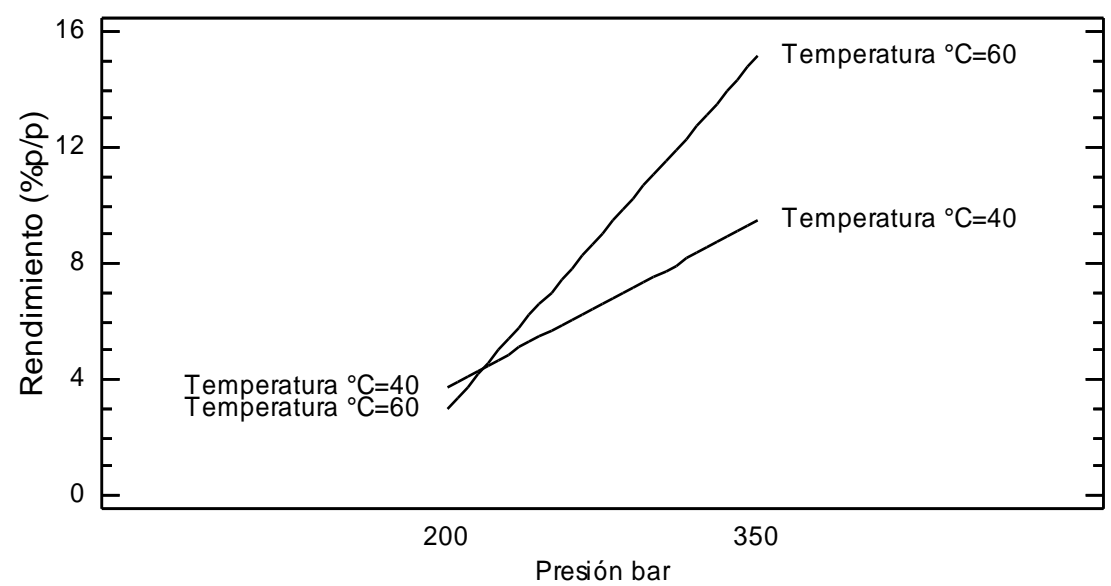

Fig. 4: Diagrama de interacción para rendimiento en la extracción de aceite de mora con $\mathrm{CO}_{2}$ supercrítico 
Se observaron rendimientos similares en la extracción de aceite de semillas de mora (Fazio et al., 2013), obtenido por el método de extracción soxhlet (15,9\%), utilizando éter de petróleo como solvente. Mientras que Pasquel et al., (2014) extrajo aceite por el mismo método, pero usando etanol, y obtuvo un rendimiento del $14,58 \%$. Otras investigaciones presentaron rendimientos de aceite diferentes, por ejemplo Cerón, et al., (2012) y García et al., (2003), obtuvieron un rendimiento del $12,2 \%$ y 9,2\% respectivamente utilizando hexano en un extractor soxhlet por 8 horas; Pasquel et al., (2014) evaluaron diferentes metodos de extracción como maceración en frio, extracción soxhlet y extracción con $\mathrm{CO}_{2}$ supercrítico. El mayor rendimiento de aceite fue de $18,25 \%$ obtenido con fluidos supercríticos. Las diferencias se deben a la técnica de extracción, tipo de solvente, condiciones de operación y principalmente a que las moras son cultivadas en diferentes zonas geográficas, suelos y otros factores que pueden influir en el contenido de aceites.

El contenido graso de semillas de mora es similar a los niveles reportados para otras semillas oleoginosas como oliva (12,37\%) extraído con $\mathrm{CO}_{2}$ supercrítico a 240 bar y $60^{\circ} \mathrm{C}$ (Al-Otoom et al., 2014), y uva con un rendimiento de aceite que oscila entre 12,35 y 16\%, obtenido por extracción soxhlet (Nilgün, et al., 2007). Estos rendimientos evidencian la posibilidad de utilizar las semillas de mora como materia prima para la obtención de aceite.

\section{Efecto de P y $T$ de extracción sobre la composición de ácidos grasos}

El análisis por cromatografía de gases con un detector de ionización de llama (GC-FID) permitió determinar que el aceite de semillas de mora está compuesto por una fracción mayoritaria de ácidos grasos insaturados tales como linoléico, linolénico y oléico, representan el $96,0 \%$ del aceite, mientras que los saturados, entre ellos palmítico y esteárico, solo representan el 4,0\%, (Figura 5). Similar composición de insaturados fue reportada por Yang, et al. (2011) y Micic et al., (2015) con el $91,2 \%$ y 93,48\% respectivamente, y con alguna variación se observa en los resultados obtenidos por Fazio et al. (2013) con un rango de 84-88,6\% y Conrado (2008) el $89,85 \%$ de insaturados.

El perfil de ácidos grasos del aceite de mora, principalmente la fracción insaturada es comparable con la composición de aceite de nuez (Martínez, et al., 2008), sacha inchi (Follegatti, 2007), ajonjolí (Botelho, et al., 2014), semillas de uva (Fiori, et al., 2014), soja, maíz (Briceño y Navas, 2005) y semillas de girasol (Rai, et al., 2016).

El ácido linoléico, es un ácido graso poliinsaturado presente en el aceite de semillas de mora en mayor porcentaje del contenido total de ácidos grasos, seguido por el ácido linolénico y en menor porcentaje los ácidos palmítico, oléico y esteárico (Tabla 1). Además se observa que la composición de ácidos grasos obtenida a alta presión y temperatura muestra un aumento en el contenido de ácido linoléico, mientras que disminuyen ligeramente los niveles de los ácidos linolénico, palmítico, oléico y esteárico. Por lo tanto las semillas de mora se pueden considerar como fuente de ácido linoléico, debido a su amplia gama de aplicaciones.

Tabla 1: Composición relativa de ácidos grasos (\%) en aceite de semillas de mora extraídos con $\mathrm{CO}_{2}$ supercrítico

\begin{tabular}{|c|c|c|c|c|c|c|}
\hline $\begin{array}{c}\text { Condición } \\
\mathrm{P}\left(\text { bar)-T }\left({ }^{\circ} \mathrm{C}\right)\right.\end{array}$ & $\begin{array}{c}\text { Ácido } \\
\text { Palmítico }\end{array}$ & $\begin{array}{c}\text { Ácido } \\
\text { Esteárico }\end{array}$ & $\begin{array}{c}\text { Ácido } \\
\text { Oléico }\end{array}$ & $\begin{array}{c}\text { Ácido } \\
\text { Linoléico }\end{array}$ & $\begin{array}{c}\text { Ácido } \\
\text { Linolénico }\end{array}$ & $\begin{array}{c}\text { Relación } \\
\text { Insa/Sat }\end{array}$ \\
\hline $200-40$ & 3,91 & 0,30 & 6,29 & 56,00 & 33,49 & 22,75 \\
\hline $200-60$ & 7,07 & 1,62 & 7,01 & 46,53 & 37,67 & 10,50 \\
\hline $275-50$ & 4,18 & 0,40 & 2,49 & 57,89 & 35,11 & 20,85 \\
\hline $350-40$ & 3,64 & 0,37 & 3,92 & 56,72 & 35,38 & 23,95 \\
\hline $350-60$ & 4,36 & 0,62 & 2,84 & 61,61 & 30,58 & 19,08 \\
\hline
\end{tabular}

\section{Efecto de la P y T de extracción sobre la concentración de escualeno, esteroles y tocoferoles}

Entre los esteroles encontrados en el aceite de mora, el $\beta$-sitosterol es el más abundante, al igual que en el aceite de semillas de uva (Fiori et al., 2014), oliva y girasol; superando al aceite de soja (Hurtado, 2002), el cual es un compuesto presente en la mayoria de semillas vegetales y parece ofrecer una seria de beneficos para la salud (Jones y Abumweis., 2009 citado por Hurtado, et al., 2014). Además en el aceite se identificaron también los esteroles 24-metil-cicloartanol y estigmasterol en menor concentración, comparable con el aceite de palma estudiado por Marrero et al., (2013). 


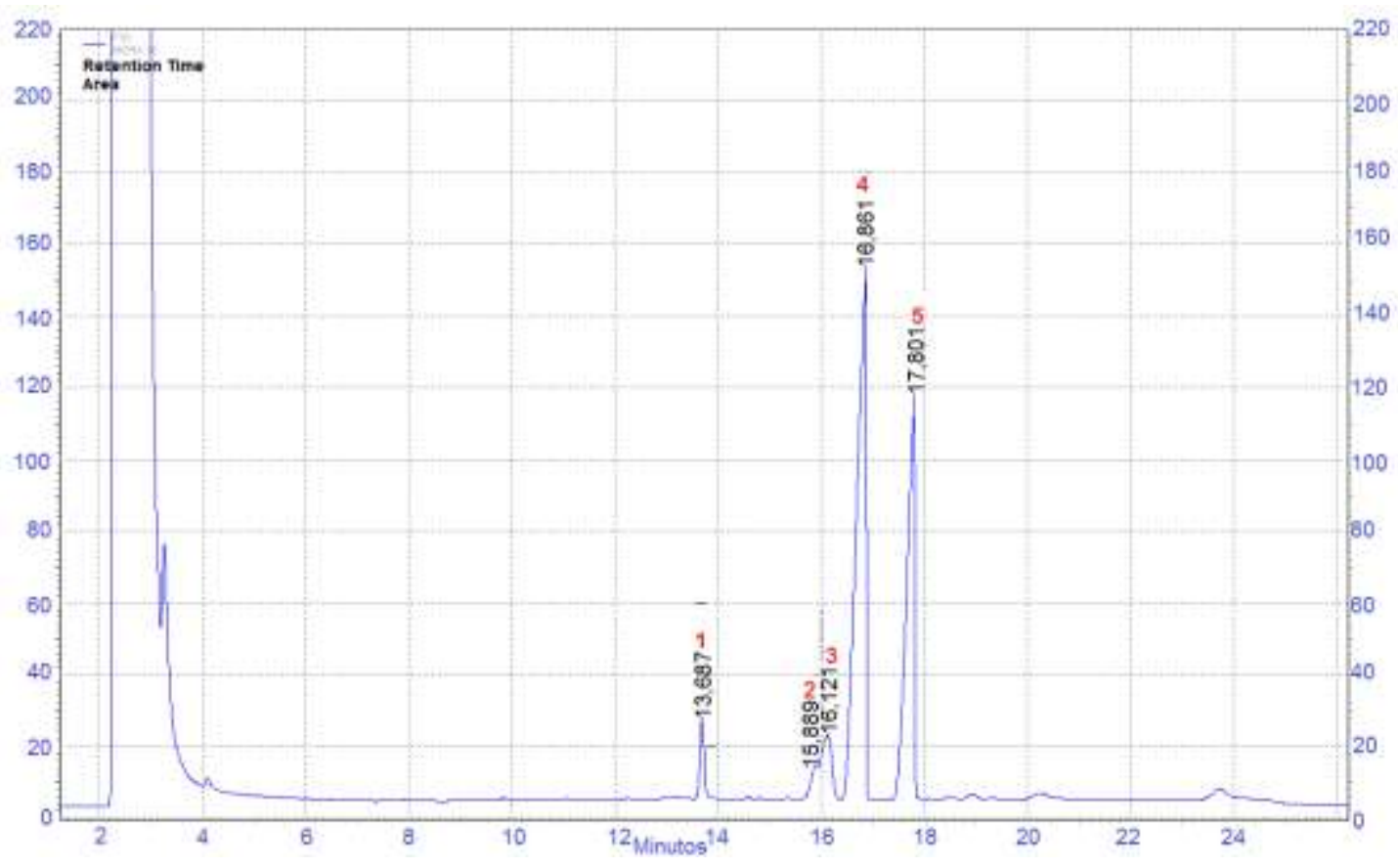

Fig. 5: Cromatograma de ácidos grasos en aceite de semillas de mora 1: Palmítico, 2: Esteárico, 3: Oléico, 4: Linoléico, 5: Linolénico

En la tabla 2 se observa que la concentración de esteroles aumenta a baja presión y alta temperatura de los niveles evaluados. Este hecho ha sido reportado por otros autores (Liu et al., 2012). El escualeno es un hidrocarburo insaturado de 30 átomos de carbono y 6 dobles enlaces, producto intermedio del colesterol, es un compuesto presente en el aceite de semillas de mora y de gran importancia en la industria alimentaria, cosmética y farmacéutica para su uso como foto protector, humectante de la piel, en la producción de alimentos funcionales, fabricación de fármacos, antioxidante natural, entre otros (Gaforio, et al., 2015); su contenido aumenta a bajas presiones (200 bar), presentando concentraciones similares a la de aceite de oliva (Hurtado, 2002) y superando a aceites de linaza, maiz, soja, girasol, algodón y cacahuete (Kiritsakis, 1992, citado por Hurtado, 2002).

Tabla 2: Contenido de escualeno y esteroles $(\mathrm{mg} / \mathrm{mL})$ en aceite de semillas de mora extraídos $\mathrm{CO}_{2}$ supercrítico

\begin{tabular}{|c|c|c|c|c|c|}
\hline $\begin{array}{c}\text { Condición } \\
\mathrm{P}\left(\text { bar) }-\mathrm{T}\left({ }^{\circ} \mathrm{C}\right)\right.\end{array}$ & Escualeno & Estigmasterol & $\beta$-sitosterol & 24-metilencicloartanol & $\begin{array}{c}\text { Esteroles } \\
\text { Totales }\end{array}$ \\
\hline & \multicolumn{5}{|c|}{$\mathrm{mg} / \mathrm{mL}$ aceite } \\
\hline $200-40$ & 1,83 & 0,08 & 4,55 & 1,03 & 5,66 \\
\hline $200-60$ & 1,30 & 0,00 & 6,28 & 1,28 & 7,55 \\
\hline $275-50$ & 0,51 & 0,08 & 4,01 & 0,90 & 4,98 \\
\hline $350-40$ & 0,35 & 0,07 & 3,20 & 0,73 & 4,00 \\
\hline $350-60$ & 0,38 & 0,00 & 3,58 & 0,78 & 4,35 \\
\hline
\end{tabular}

En la tabla 3 se presenta la concentración de los tocoferoles encontrados en el aceite de semillas de mora extraído con fluidos supercríticos. En el análisis se identificó $y$-tocoferol en mayor concentración, seguido por $a$-tocoferol y $\delta$-tocoferol. Los resultados son comparables a los reportados por Fazio et al. (2013), Yang et al. (2011) y Conrado (2008). El contenido de tocoferoles totales aumenta a bajas presiones (200 bar), que indica que el $\mathrm{CO}_{2}$ en estas condiciones puede ser más selectivo para este tipo de compuestos (Pasquel et al. (2014). Sin embargo Jokíc, et al., (2012) comenta que la concentración de tocoferoles disminuye con el aumento de la presión debido al aumento en el rendimiento total con el incremento de la presión que está relacionada con la capacidad de extracción de otros compuestos de la matriz.

La concentración de tocoferoles de aceite de semillas de mora es superior al encontrado en cualquier otro aceite reportado en la literatura, incluyendo al de oliva (Ibañez et al., 2002), palma (Gonçalves et al., 2016) y uva (Fiori et al., 2014). Además sobrepasa los niveles para aceites vegetales especificados en Codex Alimentarius (2015); lo cual hace al producto extraído, una fuente promisoria de tocoferoles naturales. 
Tabla 3: Contenido de tocoferoles $(\mathrm{mg} / \mathrm{kg})$ en aceite de semillas de mora extraídos con $\mathrm{CO}_{2}$ supercrítico

\begin{tabular}{|c|c|c|c|c|}
\hline $\begin{array}{c}\text { Condición } \\
\mathrm{P}\left(\text { bar)- }\left({ }^{\circ} \mathrm{C}\right)\right.\end{array}$ & $\delta$-tocoferol & y-tocoferol & $\alpha$-tocoferol & $\begin{array}{c}\text { Tocoferoles } \\
\text { Totales }\end{array}$ \\
\hline & \multicolumn{4}{|c|}{$\mathrm{mg} / \mathrm{kg}$ aceite } \\
\hline $200-40$ & 492,1 & 4899,4 & 941,4 & 6332,9 \\
\hline $200-60$ & 1123,2 & 8183,6 & 1315,8 & 10622,6 \\
\hline $275-50$ & 492,1 & 5134,8 & 770,2 & 6397,1 \\
\hline $350-40$ & 374,4 & 3637,1 & 534,9 & 4546,4 \\
\hline $350-60$ & 374,4 & 4150,6 & 588,4 & 5113,4 \\
\hline
\end{tabular}

En el estudio de la significancia estadística de los factores sobre el rendimiento, composición y actividad antioxidante se utilizó un análisis de varianza como se indica en la tabla ANOVA (Tabla 4). Los factores presión y la interacción presión-temperatura fueron estadísticamente significativos frente al rendimiento $(p<0,05)$; la temperatura no presentó efectos significativos sobre el rendimiento en los niveles evaluados.

En cuanto a la composición de ácidos grasos las variables independientes ejercieron un efecto significativo únicamente sobre la fracción del ácido linoléico, por cuanto el análisis de varianza muestra claramente que los factores que inciden en el contenido del ácido son la temperatura y la interacción de los factores presióntemperatura $(p<0,05)$.

Respecto al contenido de compuestos minoritarios, la presión ejerció un efecto estadísticamente significativo sobre el contenido de tocoferoles totales; mientras que la temperatura y presión tuvieron un efecto significativo sobre la concentración de esteroles totales, presentes en el aceite de mora. La interacción presión-temperatura fue el único factor significativo sobre la actividad antioxidante del aceite $(p<0,05)$.

Tabla 4. Análisis de varianza (ANOVA), del proceso de extracción de aceite de semillas de mora

\begin{tabular}{|l|c|c|c|c|c|}
\hline \multirow{2}{*}{\multicolumn{1}{|c|}{ Factores }} & \multirow{2}{*}{ Rendimiento } & \multicolumn{3}{|c|}{ Composición } & \multirow{2}{*}{$\begin{array}{c}\text { Actividad } \\
\text { Antioxidante }\end{array}$} \\
\cline { 3 - 5 } & & $\begin{array}{c}\text { Ácido } \\
\text { Linoléico }\end{array}$ & $\begin{array}{c}\text { Esteroles } \\
\text { Totales }\end{array}$ & $\begin{array}{c}\text { Tocoferoles } \\
\text { Totales }\end{array}$ & 0,7739 \\
\hline Presión & 0,0001 & 0,0605 & 0,0090 & 0,0061 & 0,1781 \\
\hline Temperatura & 0,0778 & 0,0271 & 0,0488 & 0,1163 & 0,0109 \\
\hline Interacción P-T & 0,0297 & 0,0421 & 0,1099 & 0,2597 & 0,039 \\
\hline
\end{tabular}

\section{Actividad antioxidante. Método ABTS*}

La mayor actividad antioxidante obtenida por el método $\mathrm{ABTS}^{\circ *}$ se observa en las condiciones a 200 bar$40^{\circ} \mathrm{C}$ y 350 bar- $60^{\circ} \mathrm{C}$, con valores de $604 \pm 32$ y $592 \pm 34 \mu \mathrm{mol} \mathrm{TE} / 100 \mathrm{~g}$ aceite respectivamente, como se observa en la figura 6. Esto podría explicarse que a baja presión y temperatura evaluadas se presenta mayor concentración de tocoferoles, fitoesteroles e hidrocarburos (escualeno) conocidos por ser compuestos con potencial antioxidante; mientras que a alta presión y temperatura, la actividad antioxidante se puede ver favorecida por el alto \% de ácido linoléico. Según Liu et al., (2012) la favorabilidad de la actividad antioxidante del aceite es debida al alto contenido de tocoferoles en su composición.

Los resultados de actividad antioxidante en los rangos de presión (200-350 bar) y temperatura $\left(40-60^{\circ} \mathrm{C}\right)$ evaluados oscilan entre 300 y $604 \mu \mathrm{mol}$ TE/100 g aceite, los cuales son comparados con los reportados por Yang et al., (2011) en aceites obtenidos de diferentes especies de mora, extraidos con $\mathrm{CO}_{2}$ supercrítico a 350 bar y $50^{\circ} \mathrm{C}$ presentados en la tabla 5 . En esta se observa que unicamente una de las especies supera la actividad antioxidante de la presente investigación. Además el aceite presento una actividad antioxidante similar al aceite de oliva (Sousa et al., 2015) por lo que podría tener propiedades benéficas similares.

En la figura 7 se observa el efecto de la interacción de los factores, donde muestra que el efecto de un factor (presión) depende del nivel del otro factor (temperatura). 


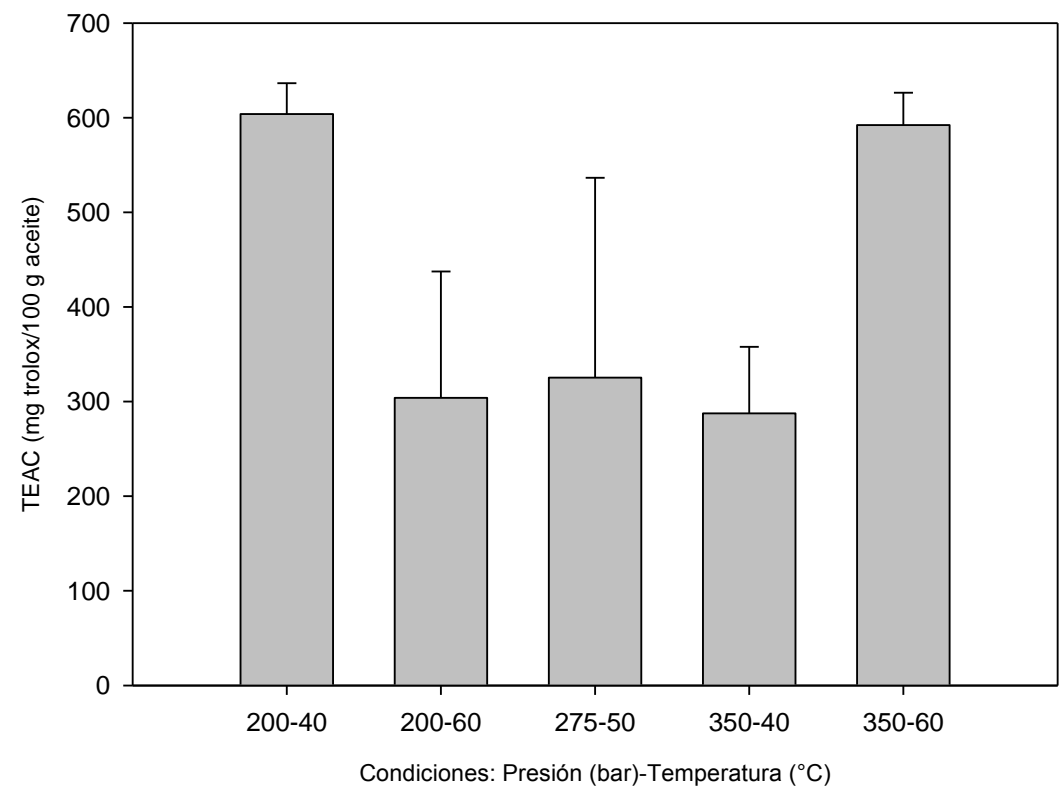

Fig. 6: Actividad antioxidante del aceite de semillas de mora extraído con $\mathrm{CO}_{2}$ supercrítico

Tabla 5: Actividad antioxidante de aceite de semillas oleoginosas

\begin{tabular}{|l|c|c|}
\hline Aceites & $\mu \mathrm{mol}$ TE/100 g aceite & Referencias \\
\hline Mora & $300-620$ & Esta investigación \\
\hline Oliva & 300 & Sousa et al., 2015 \\
\hline Arándano rojo & 338 & \multirow{3}{*}{ Yang et al., 2011 } \\
\cline { 1 - 2 } Zarzaparrilla negra & 478 & \\
\cline { 1 - 2 } Frambuesa & 424 & \\
\cline { 1 - 2 } Mora de los pantanos & 650 & \\
\hline
\end{tabular}

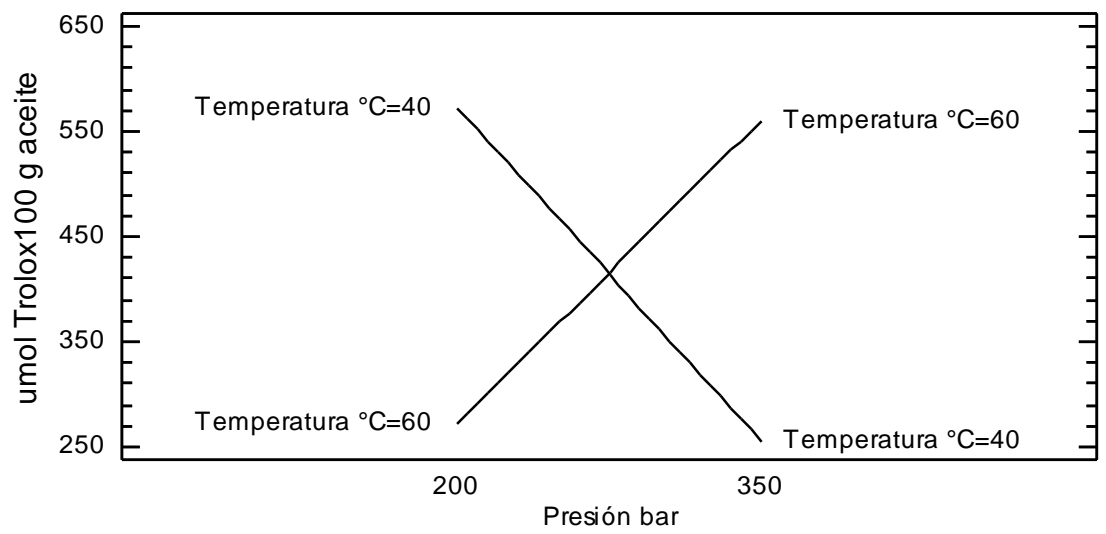

Fig. 7: Diagrama de interacciones para actividad antioxidante del aceite de semillas de mora extraído con $\mathrm{CO}_{2}$ supercrítico

\section{CONCLUSIONES}

Los resultados presentados en esta investigación demuestran que la presión y la temperatura en la extracción supercrítica mostraron efectos significativos en el rendimiento, composición y actividad antioxidante del aceite de semillas de mora. El mayor rendimiento fue $14,5 \%$, obtenido a 350 bar de presión y $60^{\circ} \mathrm{C}$ de temperatura. Los ácidos grasos insaturaturados se identificaron como componentes mayoritarios, de los cuales el ácido linoléico es el más abundante $(61,6 \%)$, seguido por linolénico, oléico y en menor 
proporción los ácidos palmítico y esteárico como ácidos grasos saturados. Además se identificó la presencia de sustancias como $\beta$-sitosterol, 24-metil-cicloartanol, $\alpha$-tocoferol, $y$-tocoferol y $\delta$-tocoferol. Las mejores condiciones para maximizar el porcentaje de ácido linoléico fueron similares a las condiciones para incrementar el rendimiento en el proceso de extracción. El aceite de las semillas de mora se constituye una alternativa de utilización de estos residuos, debido a la presencia de importantes compuestos como ácidos grasos insaturados, un alto contenido de tocoferoles naturales y gran capacidad antioxidante que lo hacen potencialmente promisorio para ser usado en la industria alimentaria, cosmética y/o farmacéutica.

\section{AGRADECIMIENTOS}

Al Fondo de Ciencia, Tecnología e Innovación del Sistema General de Regalías (SGR), a la Universidad de Nariño, a la Industria de Productos Agroindustriales de Nariño (INPADENA), a la Asociación de Productores Hortofrutícolas de Nariño (ASPHONAR) y a la Universidad Nacional de Colombia.

\section{REFERENCIAS}

Al-Otoom, A., Al-Asheh, S., Allawzi, M., Mahshi, K., Alzenati, N., Banat, B., et al. Extraction of oil from uncrushed olives using supercritical fluid extraction method. J. of Supercritical Fluids, 95, 512-518 (2014)

Botelho, J., Medeiros, N., Rodrigues, A., Araújo, M., Machado, N., Santos, A., et al. Black sesame (Sesamum indicum L.) seeds extracts by $\mathrm{CO}_{2}$ supercriticalfluid extraction: Isotherms of global yield, kinetics data, total fattyacids, phytosterols and neuroprotective effects. The Journal of Supercritical Fluids, 93, 49-55 (2014)

Briceño, J., y Navas, P. Comparación de las caracteristicas químicas, físiscas y perfil de ácidos grasos de los aceites de seje, oliva, maíz y soja. Fac. Agron, 31, 109-119 (2005)

Castro, H., Rodriguez, L., y Parada, F. Guava (Psidium guajava L.) seed oil obtained with a homemade supercritical fluid extraction system using supercritical $\mathrm{CO}_{2}$ and co-solvent. J. Supercrit. Fluids, 56 (3), 10-16. (2011).

Cavero, S., Jaime, L., Martín-Álvarez, P., Señoráns, F., Reglero, G e lbañez, E. "In vitro antioxidant analysis of supercritical fluid extracts from rosemary (Rosmarinus officinalis L.)." European Food Research and Technology 221 (3), 478-486 (2005)

Cerón, A., Osorio, O., y Hurtado, A. Identificación de ácidos grasos contenidos en los aceites extraídos a partir de semillas de tres diferentes especies de frutas. Acta Agronómica, 61(2), 126-132 (2012)

Cerón, L., Hurtado, A., y Ayala. «Efecto de la presión y la temperatura de extracción con $\mathrm{CO}_{2}$ supercrítico sobre el rendimiento y composición de aceite de semillas de guayaba (Psidium guajava).» Información Tecnológica 27, (6), En prensa (2016)

Christie, W. Gas chromatography-mass spectrometry methods for structural analysis of fatty acids. Lipids 33 , 343-353 (1998)

Codex Alimentarius. Norma para aceites vegetales especificados- Codex stan 210. Normas internacionales de los alimentos, 1-14 (2015)

Conrado, C. Análisis proximal de semillas no comunes: palma chilena (Jubaea chilensis), cilantro (Coriandrum sativum), mora (Rubus glaucus), rosa mosqueta (Rosa aff. rubiginosa) y caracterización de su aceite. Memoria, Universidad de Chile, Ciencias de los alimentos y tecnologia química, Santiago (2008)

CORPOICA. Generación de un modelo de zonificación edafoclimática y socioeconómica a nivel departamental y municipal, para la producción de mora, lulo, maracuyá, chulupa, granadilla, uva y tomate de árbol en el departamento del Huila. Informe Final de Resultados, Huila, Neiva (2007)

DANE. Encuesta Nacional Agropecuaria (2013)

Duba, K., y Fiori, L. Supercritical $\mathrm{CO}_{2}$ extraction of grape seed oil: Effect of process parameters on the extraction kinetics. Supercritical fluids, 98, 33-43 (2015)

Fazio, A., Plastina, P., Meijerink, J., Witkamp, R., y Gabriele, B. Comparative analyses of seeds of wild fruits of Rubus and Sambucus species from Southern Italy: Fatty acid composition of the oil, total phenolic content, antioxidant and anti-inflamatory properties of the methanolic extracts. Food Chemistry, 140, 817-824 (2013) 
Fiori, L., Lavelli, V., Duba, K., Charan, P., Mohamedc, H., y Guella, G. Supercritical $\mathrm{CO}_{2}$ extraction of oil from seeds of six grape cultivars: Modeling of mass transfer kinetics and evaluation of lipid profiles andtocol contents. The Journal of Supercritical Fluids, 94, 71-80 (2014)

Follegatti, L. Extracao de óleo de Sasha inchi (Plukenetia volubilis L.) com dióxido de carbono supercrítico. Campinas (2007)

Gaforio, J., Sanchez, C., Lopez, A., Ramires, M., y Warleta, F. Molecular aspects of squalene and implications for olive oil and the mediterranean diet. An evidence - based approach, 281-290 (2015)

García, D., Viloria, A., Belén, D., y Moreno, M. Características fisico-químicas y composición de ácidos grasos del aceite crudo extraído de residuos de mora (Rubus glaucus Benth). Grasas y aceites, 54(4), 259$263(2003)$

Gonçalves, C., Rodrigues, C., Marcon, E., y Meirelles, A. Deacidification of palm oil by solvent extraction. Separation and Purification Technology, 160, 106-111 (2016)

Hurtado, A. Estudio del proceso de extracción de componentes minoritarios de aceite de oliva con $\mathrm{CO}_{2}$ supercrítico en contracorriente. Tesis Doctoral, Universidad Autónoma de Madrid. Departamento de Ingeniería Química, Madrid (2002)

Hurtado-Benavides, A., Dorado, D., y Sanchez-Camargo, A. «Study of the fatty acid profile and the aroma composition of oil obtained from roasted Colombian coffee beans by supercritical fluid extraction.» The Journal of Supercritical Fluids 113, 44-52 (2016)

Hurtado, A., Martín-Álvarez, P., Vázquez, L., Reglero, G., Señoráns, F., y lbáñez, E. Optimization of Countercurrent Supercritical Fluid Extraction of Minor Components from Olive Oil. Current Analytical Chemistry, 10, 78-85 (2014)

Ibañez, E., Hurtado, A., Señoráns, F., y Reglero, G. Concentration of sterols and tocopherols from olive oil with supercritical carbon dioxide. JAOCS, 79(12) (2002)

Jimenez, J., y Villareal, J. Estudio de pigmentos tipo antocianina presentes en el fruto de motilon (Hyeronima macrocarpa). Trabajo de Grado, Universidad de Nariño, Pasto (Nariño) (2008)

Liu, G., Xu, X., Gong, Y., He, L., y Gao, Y. Effects of supercrítical $\mathrm{CO}_{2}$ extraction parameters on chemical composition and free radical-scavenging activity of pomegranate (Punica granatum L.) seed oil. Food and bioproducts processing, 90, 573-578 (2012)

Marrero, D., Rodríguez, E., González, V., y Morales, C. Determinación de esteroles en la fracción insaponificable del aceite de los frutos de la palma real cubana (Roystonea regia). Revista cubana de farmacia, 47(1), 110-116 (2013)

Martínez, M., Mattea, M., y Maestri, D. Pressing and supercritical carbon dioxide extraction of walnut oil. Journal of food engineering, 88, 399-404 (2008)

Matthaus, B., y Ozcan, M. Determination of fatty acid, tocopherol, sterol contents and 1,2- and 1,3diacylglycerols in four different virgen olive oil. Food Process Technol, 1-4 (2011)

Micic, D., Ostojic, S., Simonovic, M., Krstic, G., Pezo, L., y Simonovic, B. Kinetics of blackberry and raspberry seed oils oxidation by DSC. Thermochimica Acta, 601, 39-44 (2015)

Nilgün, B., Gülcan, Ö., y Emine, Ç. Characterization of grape seed and pomace oil extracts. Grasas y aceites, 58(1), 29-33 (2007)

Nivia, A., Castro, H., Parada, F., Rodriguez, I., y Restrepo, P. Aprovechamiento integral de la guayaba (Psidium guajava l.): i. obtención de extractos a partir de semillas utilizando como solvente $\mathrm{CO}_{2}$ supercrítico. Scientia et Technica, 33, 0122-1701 (2007)

Norhuda, I., y Jusoff, K. Supercritical carbon dioxide $\left(\mathrm{SC}-\mathrm{CO}_{2}\right)$ as a clean technology for palm kernel oil extraction. J. Biochemical Technology, 1(3), 75-78 (2009)

Pasquel, J., Da Fonseca, A., Barbero, G., Rezende, C., y Martinez, J. Extraction of antioxidant compounds from blackberry (Rubus sp.) bagasse using supercritical CO2 assisted by ultrasound. Supercritical fluids, 94 , 223-233 (2014) 
Rai, A., Mohanty, B., y Bhargava, R. Supercritical extraction of sunflower oil: A central composite design for extraction variables. Food chemistry, 192, 647-659 (2016)

Re, R., Pellegrini, A., Pannala, A., Yang, M., y Rice-Evans, C. Antioxidant activity applying an improved ABTS radical cation decolorization assay. Free Radical Biology y Medicine, 26, 1231-1237 (1999)

Ruiz, M., Urueña, M., y Martinez, J. Situación actual y perspectivas del mercado de la mora. Economic research service, 1-16 (2009)

Sousa, A., Casal, S., Malheiro, R., Lamas, H., Bento, A., y Pereira, J. Aromatized olive oils: Influence of flavouring in quality, composition, stability, antioxidants, and antiradical potential. LWT - Food Science and Technology, 60, 22-28 (2015)

Van Hoed, V., De Clercq, N., Echim, C., Andjelkovic, M., Leber, E., Dewettinck, K., et al. Berry seeds: A sourceno0f specialty oils with high content of bioactives and nutritional value. Journal of food lipids, 16, 3349 (2009)

Yang, B., Ahotupa, M., Määttä, P., y Kallio, H. Composition and antioxidative activities of supercritical $\mathrm{CO}_{2-}$ extracted oils from seeds and soft parts of northern berries. Food research international, 44, 2009-2017 (2011) 